\title{
Crystal Structure of Ipolamiide Monohydrate from Stachytarpheta indica
}

\author{
Sophon Roengsumran, ${ }^{* \dagger}$ Kasem SookKongwaree, ${ }^{*}$ Nongnuj JaIboon, $*$ Narongsak Chaichit, ${ }^{* *}$ \\ and Amorn PETSOM*
}

*Department of Chemistry, Faculty of Science, Chulalongkorn University, Bangkok 10330, Thailand

**Department of Physics, Faculty of Science and Technology, Thammasat University,

Rangsit Campus, Pathumtani 12121, Thailand

(Received June 14, 2001; Accepted February 12, 2002)

The iridoid glycosides represent a large group of cyclopentano[c]pyran monoterpenoids and are found as natural constituents in a large number of plant families. They have been shown to possess various biological activities such as antimicrobial, antitumoral, hemodynamic, choleretic, and hepatoprotective, as well as being anti-inflammatory agents. However, only few examples of crystal structures of iridoids are known: for example, loganin penta-acetate monomethyl ether bromide $^{2}$ and garilidoside. ${ }^{3}$ In the present paper, we report the crystal structure of ipolamiide (compound 1, Fig. 1) isolated previously by Tantisewie and Sticher ${ }^{4}$ from Stachytarpheta indica VAHL, which is used locally as an anti-inflammatory agent.

Ipolamiide was crystallized as monohydrate from ethanol in the orthorhombic system with space group $P 2{ }_{1} 2_{1} 2_{1}$ and $a=$ 7.885(3) $\AA, b=10.363(4) \AA$ and $c=24.498(7) \AA$. There are four formula units in one unit cell with $1.408 \mathrm{mg} / \mathrm{m}^{3}$ calculated density.

The data collection was performed on a Bruker SMART CCD area detector diffractometer. The crystal and experimental data are given in Table 1. The structure was solved by direct methods using SHELXS. ${ }^{5}$ All H-atoms except the methyl hydrogen atoms of $\mathrm{C}(12)$ were found from the difference Fourier maps and are included in the refinement with isotropic thermal parameters using SHELXL. ${ }^{5}$ The methyl hydrogen atoms of $\mathrm{C}(12)$ were included in calculated positions and were isotropically refined in a riding model. The atomic coordinates are listed in Table 2. The molecular structure and the atomic numbering are given in Fig 2. The absolute configuration has

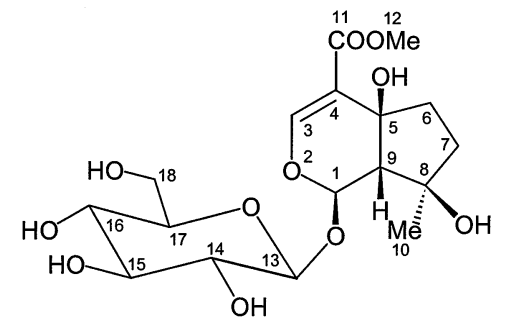

Fig. 1 Chemical structure of ipolamiide.

$\dagger$ To whom correspondence should be addressed.

E-mail: amorn.p@chula.ac.th not been determined. The enantiomorph used in the refinement was based on the known configuration of the glucosyl moiety.

In Fig. 2, the water molecule participates in a three-centered hydrogen bonding to the neighboring molecules forming a chain of molecules. The water molecule also forces the molecule to adopt a gauche conformation with indirect intramolecular hydrogen bonding between glucosyl moiety and the other unit.

The molecular packing is shown in Fig. 3. The molecules interact side by side through the interaction of hydrogen bonds of glucose unit to form a chain of molecules with the assistance of water molecules. An ipolamiide molecule is locked into position by two hydrogen bonds involving a molecule of water via $\mathrm{O}(13)-\mathrm{H}_{2} \mathrm{O}(6)-\mathrm{O}(11)$. One hydrogen atom of the water molecule forms a hydrogen bond with $\mathrm{O}(13)[\mathrm{H}(2 \mathrm{~W}) \cdots \mathrm{O}(13)$ 2.02(4) $\AA$ ], while the hydrogen atom of $\mathrm{O}(11)$ forms a hydrogen bond with the oxygen atom of the water molecule $[\mathrm{H}(11) \cdots \mathrm{O}(6)$

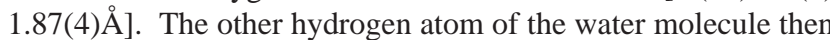
forms a hydrogen bond with $\mathrm{O}(8)$ on the glucose moiety of the second molecule $[\mathrm{H}(1 \mathrm{~W}) \cdots \mathrm{O}(8) 1.94(7) \AA]$. Thus the molecular stacking is formed along the $b$-axis.

Table 1 Crystal data and structure refinement

Formula: $\mathrm{C}_{17} \mathrm{H}_{26} \mathrm{O}_{11} \cdot \mathrm{H}_{2} \mathrm{O}$

Formula weight $=424.39$

X-rays: Mo $\mathrm{K}_{\alpha}$

Wavelength: $0.71073 \AA$

Crystal system: orthorhombic

Space group: $P 2{ }_{1} 2{ }_{1} 2_{1} \quad Z=4$

$a=7.885(3) \AA$

$b=10.363(4) \AA$

$c=24.498(7) \AA$

$V=2001.8(12) \AA^{3}$

$D_{\mathrm{x}}=1.408 \mathrm{~g} / \mathrm{cm}^{3}$

Absorption coefficient $=0.120 \mathrm{~mm}^{-1}$

Crystal size: $0.06 \times 0.15 \times 0.25 \mathrm{~mm}$

No. of reflections collected/unique $=14890 / 5686\left[R_{\text {(int) }}=0.0837\right]$

No. of reflections used $[I>2 \sigma(I)]=5686$

Refinement: full-matrix least-squares method on $F^{2}$

Goodness-of-fit on $F^{2}=1.039$

Final $R$ indices $[I>2 \sigma(I)]=R 1=0.0688, w R 2=0.0876$

$R$ indices $($ all data $)=R 1=0.1677, w R 2=0.1124$

Measurement: Bruker CCD SMART area detector

Monochromator: graphite

Structure determination: SHELXL-97 


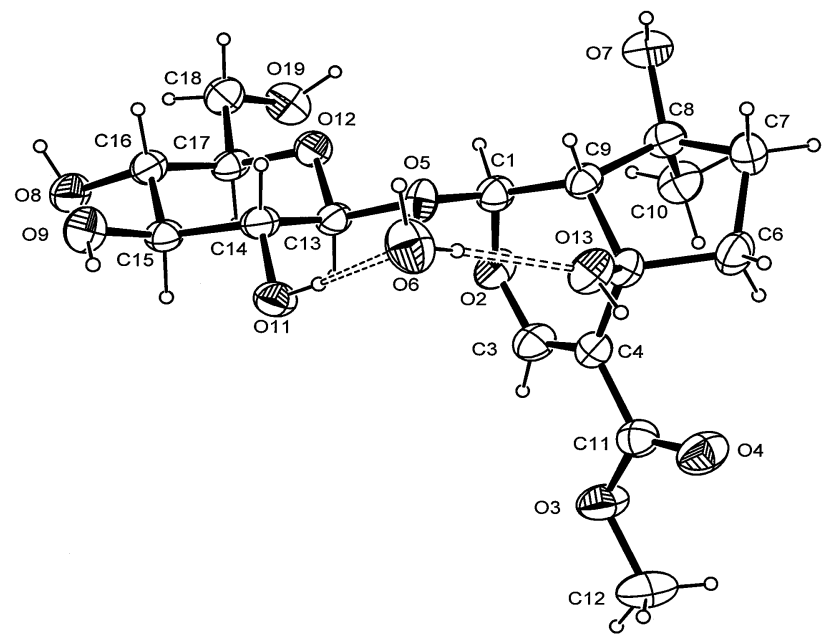

Fig. 2 An ORTEP drawing of compound 1, representing heavy atoms as $50 \%$ probability ellipsoids and $\mathrm{H}$ atoms as spheres of arbitrary radius.

Table 2 Atomic coordinates $\left(\times 10^{4}\right)$ and equivalent isotropic displacement parameters $\left(\AA^{2} \times 10^{3}\right)$

\begin{tabular}{lrrrr}
\hline Atom & $x$ & $y$ & $z$ & $U_{\text {eq }}$ \\
\hline $\mathrm{C}(1)$ & $3927(4)$ & $5287(3)$ & $5940(1)$ & $32(1)$ \\
$\mathrm{C}(3)$ & $3419(5)$ & $3039(3)$ & $5938(1)$ & $37(1)$ \\
$\mathrm{C}(4)$ & $5024(4)$ & $2713(3)$ & $6035(1)$ & $30(1)$ \\
$\mathrm{C}(5)$ & $6436(4)$ & $3701(3)$ & $6021(1)$ & $29(1)$ \\
$\mathrm{C}(6)$ & $7848(5)$ & $3358(3)$ & $5606(2)$ & $39(1)$ \\
$\mathrm{C}(7)$ & $8141(5)$ & $4575(3)$ & $5262(2)$ & $39(1)$ \\
$\mathrm{C}(8)$ & $6365(4)$ & $5163(3)$ & $5226(1)$ & $34(1)$ \\
$\mathrm{C}(9)$ & $5773(4)$ & $5026(3)$ & $5826(1)$ & $29(1)$ \\
$\mathrm{C}(10)$ & $5259(6)$ & $4461(4)$ & $4815(2)$ & $41(1)$ \\
$\mathrm{C}(11)$ & $5444(4)$ & $1346(3)$ & $6120(1)$ & $33(1)$ \\
$\mathrm{C}(12)$ & $4386(5)$ & $-796(3)$ & $6169(2)$ & $56(1)$ \\
$\mathrm{C}(13)$ & $2170(4)$ & $5771(3)$ & $6709(1)$ & $28(1)$ \\
$\mathrm{C}(14)$ & $2329(4)$ & $6083(3)$ & $7307(1)$ & $30(1)$ \\
$\mathrm{C}(15)$ & $590(4)$ & $6396(3)$ & $7540(1)$ & $28(1)$ \\
$\mathrm{C}(16)$ & $-303(4)$ & $7417(3)$ & $7207(1)$ & $31(1)$ \\
$\mathrm{C}(17)$ & $-275(4)$ & $7061(3)$ & $6600(1)$ & $29(1)$ \\
$\mathrm{C}(18)$ & $-944(5)$ & $8126(3)$ & $6238(2)$ & $40(1)$ \\
$\mathrm{O}(2)$ & $2816(3)$ & $4234(2)$ & $5811(1)$ & $38(1)$ \\
$\mathrm{O}(3)$ & $4078(3)$ & $578(2)$ & $6133(1)$ & $46(1)$ \\
$\mathrm{O}(4)$ & $6873(3)$ & $938(2)$ & $6164(1)$ & $46(1)$ \\
$\mathrm{O}(5)$ & $3810(3)$ & $5576(2)$ & $6502(1)$ & $32(1)$ \\
$\mathrm{O}(6)$ & $6346(3)$ & $5093(3)$ & $7538(1)$ & $46(1)$ \\
$\mathrm{O}(7)$ & $6366(3)$ & $6497(2)$ & $5058(1)$ & $42(1)$ \\
$\mathrm{O}(8)$ & $-2030(3)$ & $7496(2)$ & $7388(1)$ & $44(1)$ \\
$\mathrm{O}(9)$ & $720(3)$ & $6829(2)$ & $8092(1)$ & $40(1)$ \\
$\mathrm{O}(11)$ & $2990(3)$ & $5024(2)$ & $7610(1)$ & $34(1)$ \\
$\mathrm{O}(12)$ & $1443(3)$ & $6839(2)$ & $6427(1)$ & $33(1)$ \\
$\mathrm{O}(13)$ & $7148(3)$ & $3890(2)$ & $6557(1)$ & $37(1)$ \\
$\mathrm{O}(19)$ & $-1159(4)$ & $7718(2)$ & $5686(1)$ & $46(1)$ \\
\hline & & & & \\
\hline
\end{tabular}

$U_{\text {eq }}$ is defined as one third of the trace of the orthogonalized $U_{i j}$ tensor.

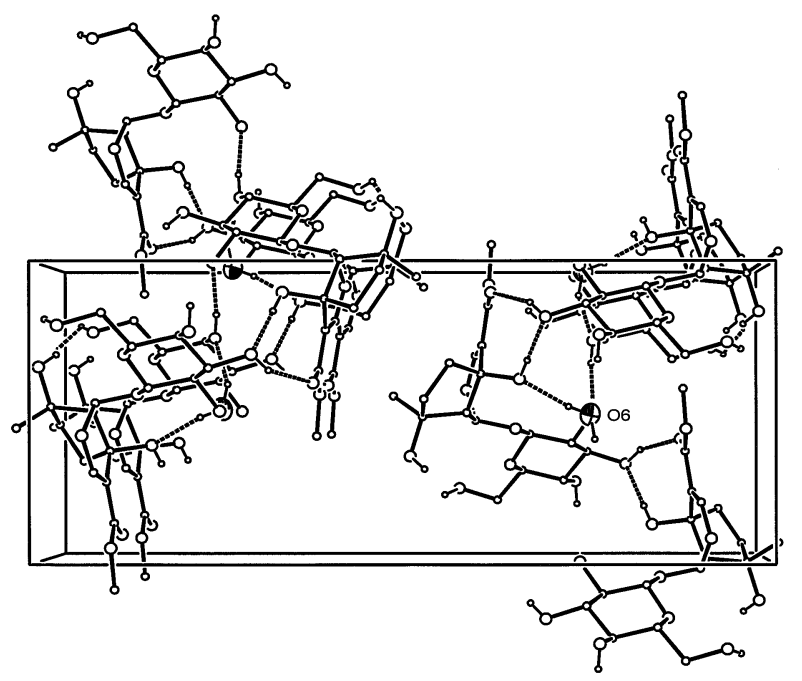

Fig. 3 A perspective drawing of the packing arrangement of compound $\mathbf{1}$ in the crystal.

Table 3 Hydrogen bonds [ $\left[\AA\right.$ and ${ }^{\circ}$ ]

\begin{tabular}{lcccr}
\hline \multicolumn{1}{c}{$\mathrm{D}-\mathrm{H} \cdots \mathrm{A}$} & $\mathrm{d}(\mathrm{D}-\mathrm{H})$ & $\mathrm{d}(\mathrm{H} \cdots \mathrm{A})$ & $\mathrm{d}(\mathrm{D} \cdots \mathrm{A})$ & $<(\mathrm{DHA})$ \\
\hline $\mathrm{O}(6)-\mathrm{H}(2 \mathrm{~W}) \ldots \mathrm{O}(13)$ & $0.79(4)$ & $2.02(4)$ & $2.780(4)$ & $163(4)$ \\
$\mathrm{O}(6)-\mathrm{H}(1 \mathrm{~W}) \ldots \mathrm{O}(8) \# 1$ & $0.94(7)$ & $1.94(7)$ & $2.823(4)$ & $155(5)$ \\
$\mathrm{O}(8)-\mathrm{H}(8 \mathrm{H}) \ldots \mathrm{O}(11) \# 2$ & $0.85(4)$ & $1.88(4)$ & $2.728(3)$ & $170(3)$ \\
$\mathrm{O}(19)-\mathrm{H}(19 \mathrm{H}) \ldots \mathrm{O}(7) \# 3$ & $0.92(4)$ & $1.91(4)$ & $2.820(4)$ & $167(3)$ \\
$\mathrm{O}(9)-\mathrm{H}(9 \mathrm{H}) \ldots \mathrm{O}(4) \# 4$ & $0.88(4)$ & $1.96(4)$ & $2.788(4)$ & $156(3)$ \\
$\mathrm{O}(7)-\mathrm{H}(7 \mathrm{H}) \ldots \mathrm{O}(19) \# 1$ & $0.89(4)$ & $1.90(4)$ & $2.789(4)$ & $176(3)$ \\
$\mathrm{O}(11)-\mathrm{H}(11 \mathrm{H}) \ldots \mathrm{O}(6)$ & $0.79(4)$ & $1.87(4)$ & $2.653(4)$ & $172(4)$ \\
$\mathrm{O}(13)-\mathrm{H}(13 \mathrm{H}) \ldots \mathrm{O}(9) \# 5$ & $0.83(4)$ & $2.02(4)$ & $2.850(3)$ & $175(4)$
\end{tabular}

Symmetry transformations used to generate equivalent atoms: \#1 $x+$ $1, y, z ; \# 2-x, y+1 / 2,-z+3 / 2 ; \# 3 x-1 / 2,-y+3 / 2,-z+1 ; \# 4-x+1$, $y+1 / 2,-z+3 / 2 ; \# 5-x+1, y-1 / 2,-z+3 / 2$.

\section{Acknowledgements}

The financial support of the National Research Council of Thailand is gratefully acknowledged.

\section{References}

1. M. D. C. Recio, R. M. Giner, S. Manez, and J. L. Rios, Planta Med., 1994, 60, 232.

2. P. J. Lentz, Jr. and M. G. Rossmann, Chem. Commun., 1969, 1269.

3. D. Tasdemir, L. Scapozza, O. Zerbe, A. Linden, I. Calis, and O. Sticher, J. Nat. Prod., 1999, 62, 811.

4. B. Tantisewie and O. Sticher, Phytochemistry, 1975, 14, 1462.

5. G. M. Sheldrick, SHELXL97, University of Göttingen, Germany, 1997. 